MedieKultur | Journal of media and communication research | ISSN 1901-9726

Article - Open section

\title{
Making sense of the German Wikipedia community
}

\section{Rikke Frank Jørgensen}

MedieKultur 2012, 53, 101-117

Published by SMID | Society of Media researchers In Denmark | www.smid.dk The online version of this text can be found open access at www.mediekultur.dk

This article presents the findings from a qualitative study of the German Wikipedia community, focusing on how people engaged with Wikipedia make sense of norms, collaborative practices and means of regulation within the community. The study highlights the strong focus on the quality of the end-product (the encyclopedia) in the German community, stressing that article quality is seen as more important than the wiki-process as such. As the community has grown, an increasing number of rules and mechanisms have been deployed to resolve various issues and conflicts, however the interviewees do not perceive Wikipedia as being bureaucratic, but rather describe it as a "rule-governed anarchy". The findings suggest that people contribute for a variety of reasons, yet point to reactions from and interactions with fellow Wikipedians as one of the strongest motivational drivers for participation.

\section{Introduction}

Over the past ten years, numerous articles and books have addressed various aspects of Wikipedia as a research topic (Bruns, 2009; Lih, 2009; Reagle, 2010), and several scholars have referred to Wikipedia as an example of so-called "free culture" due to its open and participatory format (Lessig, 2004; Benkler, 2006; Sunstein, 2006; Zittrain, 2009). Further it 
has been suggested that the "success" of Wikipedia testifies to the power of norms such as openness and sharing.

In the current study, I investigate some of these norms related to free cultures, focusing on three main themes within the Wikipedia community: 1) the values and sense of belonging emphasized by the interviewees (community culture) (McMillan \& Chavis, 1986); 2) the specific ways in which members of the community organize their contributions towards a common goal, hence the day-to-day collaborative practices of producing Wikipedia articles (London, 1995); 3) the self-regulation, referring to the rules and mechanism used to regulate behavior within the community (Schultz \& Held, 2001). In summary, the goal of the study is to understand in more detail the values and practices that constitute a particular open culture community. As my unit of analysis, I examine the German subdomain of Wikipedia, specifically the community in and around Berlin. As such, the findings represent the reflections of a small group of people (compared to the 1.3 million registered users of the German Wikipedia), however all of the interviewees have been deeply involved in Wikipedia for several years (more below). With regard to the English language edition of Wikipedia, I have included it here as a point of reference due to its status as the first Wikipedia domain, and since many of the principles and guidelines used in the German subdomain derive from the English language edition of Wikipedia. I have not intended this to be a comparative study between the two editions.

\section{Methodology}

The data collection was based on the large amount of text that the site in itself represents, and on seven qualitative interviews with people from the Berlin Wikipedia community, as well as a member of the Wikipedia Advisory Board. The interviewees were recruited with a view to ensure awareness of community norms and active participation in Wikipedia. The interviewees were identified by asking around in the community, and contacting people who were recommended as being engaged and knowledgeable Wikipedians. All of those interviewed are experienced Wikipedia editors and several of them have been active in Wikipedia policy spaces, nationally as well as internationally. As such, the interviewees represent people who have been active in Wikipedia more or less since the beginning, and several of the interviewees have held positions of trust within the community. All interviews were conducted in English. The data that are analyzed in the study are a mixture of transcribed interviews, notes, various materials presented on the Wikipedia platform, a few formal presentations, e.g., by Wikipedia founder Jimmy Wales, and some informal conversations. For the purpose of citations, the quotes are presented in "written language style", thus pauses and self-correction have been removed. Removal of text within a quote is marked by [...]. The data analysis involved a combination of meaning condensation/coding and narrative structuring, employing a theme-based approach (Thagaard, 2004, pp. 158-163). The approach was relatively bottom-up, as I allowed the actors' experiences and interpre- 
tations of practice to guide the analyses. The analyses involved identifying theme-related points from the interview scripts and drawing out relevant examples and arguments. This included sub-themes, such as why people participate in Wikipedia, how the daily collaborations between editors function, the role of the community in defining and implementing rules, and the relation between the Wikipedia community and public life more generally.

\title{
The history of the German Wikipedia
}

The German Wikipedia was announced by Jimmy Wales in March 2001, as the first nonEnglish Wikipedia subdomain. As of February 2012, the German subdomain contains more than 1.3 million articles and is the second largest next to the English domain. German Wikipedians have played an active role in the international Wikipedia community from early on and continue to be represented on the international board.

In 2003, the German community organised the first physical German Wikipedia meeting in Munich, and soon monthly "Stammtisch" (literally "a table for the regulars") became a common meeting point for the community. The stammtisch would usually have twenty to thirty people attending to debate issues such as how people behaved online, how to ban people, how to protect articles, etc. (German Wikipedian \#3).

In 2004, the community established the national chapter of Wikimedia; Wikimedia Germany. The German Wikipedia had a strong rate of growth in the first years, especially in 2004. Since mid-2006, the level of contributions has been approximately 400 new articles per day and between 6500 and 8500 active Wikipedians per month, with a slightly declining trend in 2011. ${ }^{2}$ In February 2012, the German Wikipedia had a total of 1,376,769 registered users. The exponential growth of new users in 2004 is explained by massive coverage in mainstream media.

\begin{abstract}
It was a series of fortunate events that put us into the most read newspaper, the most read news magazines, TV channels. I think it was a matter of 2 or 3 weeks when Wikipedia was extremely prominent on almost every channel and in this time the German Wikipedia increased $1 \%$ per day for almost 2 weeks and we were completely exhausted and unable to assemble the incoming traffic. I think half of the people I know jumped on Wikipedia in this period of time. So it was an artificial growth, which was unique to the German encyclopedia (German Wikipedian \#5).
\end{abstract}

In 2005, the German community organized the first global Wikimania conference in Frankfurt with 380 people from over 50 countries in attendance. ${ }^{3}$ The following year, the Göttingen State and University Library held a special exhibition documenting the first five years of Wikipedia. Also, the first two Wikipedia Academies were held in Germany in 2006 and 2007, and in 2008 the German Wikipedia was ranked higher than any other domestic news site within Germany in terms of web traffic. In May 2011, the German Wikipedia ranked number 6 amongst most visited websites in Germany. ${ }^{4}$ 
The quality of the German Wikipedia has been studied and compared to other encyclopedias several times. In December 2007, the German magazine Stern published the results of a research-based comparison between German Wikipedia and the online version of Brockhaus Enzyklopädie (Stern.de, December 5, 2007). The comparison included 50 articles from each encyclopedia and the study showed that Wikipedia articles were evaluated as being more accurate on average. Wikipedia's coverage was also found to be more complete and up to date. However, Brockhaus was judged to be more clearly written, with several Wikipedia articles being criticized for their length and complexity. In January 2011, Sue Gardner, Executive Director of the Wikimedia Foundation, stated that the German Wikipedia is "the best" language edition.

\footnotetext{
It's accurate, it's comprehensive, it's well maintained, the articles are longer, the articles are well referenced, and so forth. Germany is a wealthy country. People are well educated. People have good broadband access. So the conditions for editing Wikipedia are there. And the fact that German people were able to meet face to face and talk about policies and talk about procedures and so forth because they're geographically located in a relatively small area, for the most part (Gardener quoted in On The Media January 14, 2011).
}

These characteristics are further explored below, starting with the theme of community culture.

\section{Community culture}

Wikipedia has a set of declared principles, which have often been emphasized as a crucial normative baseline for the project to succeed. When interviewing people in the German community, I was particularly interested in how the interviewees reflected on these norms and values, and what role they played for people's decision to take part in the project.

The principles are summarized in five pillars that apply to all language editions: ${ }^{5} 1$. Wikipedia is an online encyclopedia (value of neutrality); 2 . Wikipedia is written from a neutral point of view (value of neutrality); 3. Wikipedia is free content that anyone can edit, use, modify, and distribute (value of openness and sharing); 4. Editors should interact with each other in a respectful and civil manner (value of trust and dialogue); and 5. Wikipedia does not have firm rules (value of dialogue). Further, is it stressed that Wikipedia is "more than just an encyclopedia, Wikipedia is a community".6

The first and second pillars stress that Wikipedia is an encyclopedia written from a neutral point of view (NPOV). This implies all Wikipedia articles must represent fairly, and as far as possible without bias, all significant views that have been published by reliable sources. The study found that neutrality is seen as a community-internal standard, towards which the quality of articles may be judged, rather than a value of neutrality as such. It also stressed that an article may be biased when first proposed, but the more editors work on 
it, the more neutral it becomes. Further, any national edition is likely to have content biased towards specific national topics.

Whereas the norm of neutrality addresses the way articles are written, the fourth and fifth pillars address cooperation within Wikipedia, based on dialogue and trust. As a norm for this trustful dialogue, a principle of good faith has been stipulated in the guidelines for Wikipedia editors. The good faith principle encourages Wikipedians to trust one another as sensible human beings, and to use dialogue to reach agreement via trusted communication rather than to suspect bad motives from fellow editors.

In relation to dialogue and trust, the interviewees stressed that the debate amongst the German community is harsh, whereas the English counterpart is characterized more by a be-nice and wiki-love culture (German Wikipedian \#1).

This is the official version by Jimmy Wales (wiki-love), which he always speaks about, and I think that it is not the case. I think that the principal of the German Wikipedia is, yeah, if you take a look at the community there are a lot of arguments, a lot of dispute and it is not just dispute it is really discussion and sometime it is really offensive, people offend each other very easily on the discussion page, which can sometimes be a problem because this is public and you can always see that this and that person have a very offensive way of discussing with other people so this is an interesting aspect I think [...]. If you read discussion pages you almost never get a feeling of harmony and love. It is just the opposite (German Wikipedian \#4).

It is also stressed that the relatively harsh tone in the debate might be a Western discussion culture more generally, rather than a German characteristic per se. There is thus some discrepancy between the declared principles of a kind and trustful dialogue, and the actual way of debating and disagreeing within the German community.

The third pillar is related to the licensing model, implying values of openness and sharing. On a legal level, the licensing model makes any contribution available for others to use, and Wales has argued that precisely this aspect of free licensing is empowering to people working on Wikipedia, as it ensures that the contributions belong to the broader public and not to any individual or company (Lih, 2009, pp. xvi-xvii). The study confirmed the importance assigned to the open licensing model and the practice of not claiming ownership over individual contributions. Further, it was stressed that sharing and feedback are key factors for contributing, as addressed below.

When Wikipedia was launched in Germany, many Wikipedians perceived it as being for idealists, and several were skeptical as to whether it could work in the real world. The community assembled only a handful of people at that time, and the content in the German edition was scarce and with lots of mistakes. When asked about the reasons for joining Wikipedia, the interviewees stressed that several people from the first Wikipedia generation were there for ideological reasons. Many perceived Wikipedia as a proponent for a new kind of enlightenment, building on Habermas' ideal of rational-critical conversations 
and the power of the better argument. In addition, as the content in the German edition was limited, it was relatively visible and rewarding within the community to contribute new articles.

The study found four main reasons for participating in Wikipedia, while recognising that the attempt to categorise motivations may oversimplify what might be much broader in complexity. The first theme relates to ideological reasons, explained as support for the principles on which Wikipedia is built, and a willingness to help make the world a better place, based on the principles of free knowledge.

So I think in the beginning you needed some kind of Weltverbesserungsantrieb, yeah to make the world a better place [...] I think the first generation of contributors mainly did it because of ideological reasons, like I think many people saw it as a new way of developing a new kind of enlightenment, so I am talking about my own motivation. I think this is the main reason why I was so hooked in the beginning (German Wikipedian \#6).

Most of those interviewed mentioned the ideological point of departure as a motivating factor when becoming involved with Wikipedia. However, while the ideological reasons were mentioned by practically all those interviewed, they were mostly presented in combination with some of the reasons below.

The second theme relates to the ability to express oneself and get reactions within a specific area of interest. For many contributors, Wikipedia seems to be about one or more particular interests, and the possibility of exercising that specific interest while interacting with like-minded people. The importance of being read and receiving a reaction in response to your writing are stressed time and again, precisely because they imply an active and largely qualified reaction to some of the issues that the contributor is occupied with. One of the interviewees compares this reaction to a digital hug.

That was really the thing that fascinated me from the beginning when I started to work on Wikipedia. I just could not wait for the reaction. I wrote something and I could not get away from the computer, because if I was away I was waiting for people to comment or to change my things and to react to it. And I was really keen on that, still I am keen on that reaction, it is even better now (German Wikipedian \#4).

A third and closely related theme is that of the visibility that the site represents. Not only do contributors receive reactions from people interested in the same topics, but their writings also become visible to a wider audience. Visibility is stressed as a key motivating factor in contributing to Wikipedia, however an anonymous form of visibility. It is described as being recognised in front of a wider audience for the contributions made, but without the usual link to physical identity, name, and status. Some of the interviewees go as far as to describe these two factors, interaction and visibility, as a way out of loneliness.

Fourth, the community involvement is mentioned as an important driver for taking part in Wikipedia. Whereas the ideological focus is mostly mentioned as a factor when deciding 
to join Wikipedia, the sense of being part of a group of like-minded people working on a common goal is emphasized time and again. As illustrated by the quote below, there is a clear distinction between the community itself and public life as such, with the latter receiving less attention.

\begin{abstract}
It is a bit irritating how important it is for them to be recognized inside the community and how unimportant it is for many people to know about millions of readers that are out there $[\ldots]$ but the thing that people realize when some community member leaves a comment on your discussion page and says you have written a great article, there just two things I did not understand and so forth, I have some sources here that may be interesting for you [...] this is the feed back that people see and it motivates them much more than just some numbers where you don't have faces (German Wikipedian \#6).
\end{abstract}

Finally, some of the interviewees mention that besides the reasons discussed above, they simply like writing and editing, and enjoy solving various problems that occur in the process. Also, it was stressed that they are proud to be part of the project.

Thus, the way the interviewees describe the German community by and large reflects Wikipedia's founding principles and values. However, it nonetheless differs on certain aspects. The most notable difference is related to the dialogue between editors, which is described as relatively harsh compared to the norm of a trustful dialogue based on good faith. In relation to participation, the sense of being part of a larger vision, with which one agrees, is seen as important for the willingness to contribute as well as the reward of exchange and feedback from people with similar interests. The contributors are aware of, and support, the founding vision of Wikipedia. Yet in their daily practices, they seem less occupied with the normative goal (to set knowledge free), and are more oriented towards writing and exchanging views in a dialogue with other community members. Responses to one's own writings by other Wikipedians with similar interests are mentioned as one of the strongest drivers for participation. In this regard, the norm of sharing seems particularly important for taking part in Wikipedia.

\title{
Collaboration
}

As for the second theme, collaboration, I was particularly interested in how the above-mentioned values are reflected in the practices that constitute cooperation within Wikipedia.

As stressed by the study, collaboration within Wikipedia is essentially about co-production in which groups of people voluntarily cooperate on a common product, with each article being the result of a collective process. In practice, the day-to-day activity of creating and editing Wikipedia articles is done in smaller units, gathered around specific fields of interests.

The collaborative model is anchored in the Internet's open and decentralised architecture, which in principle allows for cooperation amongst anyone connected. This decentral- 
ised collaboration on a shared resource would thus not be possible within the context of conventional media. With regard to values, the collective process requires openness and sharing in the sense that any contribution must be open for other people to comment on and to further refine.

One of the essentials of the collaboration is the production of cumulative knowledge, whereby people gradually improve each other's work and, in this way, create a totality that no one could accomplish alone. In practice this is achieved via so-called stubs, which are preliminary article drafts. As explained below, stubs are not allowed in the German edition, thus collaborative work on new articles starts at a more elaborated level compared to the English edition. When asked about the contributors' sustained willingness to improve the common product, it was stressed that some articles are more popular than others and subject to more attention from fellow editors. In other words, peer assistance to improve articles may not always occur, just as some articles may receive a disproportionate amount of attention simply because the theme is a hot topic. "So if a page becomes more popular, the chances that someone else will improve the article just because it is popular are going to increase" (German Wikipedian \#5).

Another characteristic is the voluntary character of the collaboration. Due to the voluntary nature of the work, there is no guarantee that people will respond to the writing done by any given editor, however if people were not generally willing to respond to work initiated by fellow Wikipedians, Wikipedia would cease to exist. "There is a proverb thing like 'people vote with their feet', so they decide where to work, so if they are there, it contains the answer. I think" (German Wikipedian \#5). As a way of encouraging various contributions that are seen as essential to the German edition, the community since 2005 has had a reward mechanism, hence a page where users may offer rewards (Auftragsarbeiten) for completion of Wikipedia-related tasks. The reward mechanism is an example of soft intervention in the voluntary process. Moreover, the study found that despite an emphasis on sharing, there is an expectation of "pay back" from people who have benefited from Wikipedia. One of the interviewees argued that by now many people have benefited from Wikipedia for a longer period of time, hence they have a moral obligation to start giving something back to the common pool of knowledge (German Wikipedian \#5).

In relation to disagreements between editors, the community entails several dialogueoriented spaces that facilitate disagreement and provide for meta-debate. These spaces include "talk pages", the "articles for deletion" forum (a common space in which articles proposed for deletion are debated), and a number of mailing lists. In relation to the dayto-day editing of articles, the term edit war is used to coin the conflicts that unfold around the collaboration on specific articles.' In an edit war, contributors disagree on the substance of a given topic and typically keep reverting the page to a version they approve of or mark the page as one that ought to be deleted. As a means of creating openness around the conflicts, the article history is stressed as an essential feature making the differences in opinions transparent, and illustrating the conflicting viewpoints in the various article versions. 
However, as stressed by one of the interviewees, there are many cases in which it is difficult to resolve which argument should prevail in the article in question. "There is still no really good method to deal with two qualified opinions" (German Wikipedian \#1).

With regard to collaboration within the German community, one of the themes that kept surfacing relates to the approach towards the end product vis-à-vis the process itself. According to the interviewees, the English-speaking community is oriented towards the process, i.e., the collaborative principles of the wiki platform and the open and inclusive way of cooperating on a common text (the "wiki way"); whereas the German community is more focused on the end product, producing the best encyclopedia ever.

We are not here because we want to use the wiki and have fun with it, but we want to have an encyclopedia which is bigger and better than any encyclopedia that has been there before [...] they say it is not the 'reine Lehre' the original idea of the wiki as such. But I would say and I think many people in the German community also would say the wiki is just a tool and if we have another tool which is better for writing an encyclopedia we will use that and we are not bound to use the wiki forever (German Wikipedian \#6).

The importance of article quality is manifested in a system of stable versions, deployed by the German community. The feature of stable versions implies that certain users are able to mark article versions as "reviewed", indicating that the text contains no obvious vandalism. The idea of these flagged revisions is to indicate that a given text has been validated by Wikipedians according to certain standards of quality (spelling, factual accuracy, etc.), allowing for work-in-progress on a given article "behind the scenes", while presenting the validated version to the public. The German community was first to introduce stable versions in 2008, and paid developers to deploy it on the German Wikipedia.

Also, the issue of article relevance is a crucial topic within the German community, and is referred to as the "excludist vs. includist" approach by those interviewed. The debate on article relevance relates to the emphasis on the quality of the final product, rather than letting everyone contribute in a more inclusive manner.

There are two groups, and this is I think very typical again of the German Wikipedia and not as visible in the other Wikipedias although it is present everywhere [...] the includists and the excludists. The excludists want to take out things because they think they are not relevant and the includists want to put everything in. [...] and there is a lot of discussion about relevance and perhaps such discussion is a good thing because those who want to keep the article have to improve it so the discussion can foster the article (German Wikipedian \#4).

As an indicator of the German focus on quality, the German guidelines classify scholarly sources as inherently more reliable than non-academic sources, and articles on "indisputably notable subjects" may be deleted if they are deemed too short. Consequently it has been decided to eliminate the category "stub" from the German Wikipedia and, unlike many 
other Wikipedias, the German edition does not contain large collections of bot-generated geographical stubs or similar articles.

Another feature of the German community is the extensive use of discussions within the community, as pointed to by several of the interviewees. It is stressed that the voluntary character of the work in principle provides unlimited time to discuss, contrary to organizations with commercial agendas. "We are free to discuss things forever; as long as we don't have really big juridical problems, there is nothing that forces us to be in a hurry. So this is a very comfortable position that we are in" (German Wikipedian \#6).

As a final characteristic of collaboration in the German community, the study highlighted the large amount of offline activities, e.g., study tours and visits to other Wikipedia communities nationally and abroad (e.g., to the Balkans), city walks to explore potential Wikipedia articles, and various projects that link Wikipedia articles with physical sites.

In summary, Wikipedia is based on collaborative processes, where numerous individuals contribute to the final product, organized in sub groups around specific articles. The collaborative model is based on commons (shared resources) and is voluntary, as no employment structure is involved. The day-to-day work on Wikipedia is thus highly dependent on people's willingness to share their work, to contribute to the work of others, and to invest time without any monetary compensation. The study found that the specifics of the German community relate to a strong focus on the quality of the final product vis-à-vis the wiki process. Also, long lasting discussions among community members were emphasized as a characteristic of the community's way of cooperating. In relation to values, the focus on the quality of the German encyclopedia implies that the community has defined limits to its openness, such as restrictions on which articles to include. Finally, with regard to sharing, people are to some extent expected to pay back to the community.

\section{Self-regulation}

The final theme, self-regulation, refers to the rules and procedures that regulate behavior within the community.

The regulatory model of Wikipedia has been described as a light regulatory touch coupled with an openness to flexible public involvement, including a focus on earnest discussion, neutral dispute resolution, and a "core of people prepared to model an ethos, which others can follow" (Zittrain, 2009, p. 146). Wikipedia presents itself as a deliberative democracy where discussion amongst community members is used as the primary means to reach agreement, rather than votes. ${ }^{8}$

Despite the claim for a light regulatory model, a remarkable amount of written guidelines, policies, official positions, and mechanisms to counter various community problems are actually in place, not least if compared with conventional grassroots organizations. When asked whether Wikipedia has turned into a bureaucracy, the interviewees stated that though the German Wikipedia is more regulated than the English counterpart, Wikipedia is 
not a bureaucracy but rather a mixture of community anarchy and community rules. "Yes it (the community) is anarchy-based. There are rules, there are normal Wikipedians, there are the administrators and that is it. There are bureaucrats but they are very few very few" (German Wikipedian \#6). Below I address some of the positions and mechanisms incorporated as part of the community rule.

To start with, there is the so-called "constitutional monarch" of English-speaking Wikipedia, Jimmy Wales, who is described as someone who still appears to have much weight in the international community due to his special role as Wikipedia founder. It is emphasised that Wales is aware of his special position and takes an accordingly diplomatic stand in discussions.

He [Jimmy Wales] is still active in large debates. He is aware of his reputation, if he speaks out on some issue, people will take notice of this statement, so which makes his statements in many ways usually more balanced, diplomatic than they could be. [...] he is an authority thanks to his role in starting the whole Wikipedia (German Wikipedian \#5).

Wales is chairman emeritus of the Wikimedia Board of Trustees, which is the ultimate corporate authority in the Wikimedia Foundation. However, despite Wales' status and recognition, it is stressed that his monarchical power is inherently dependent on the consent of the community (German Wikipedian \#1).

With regard to the Wikimedia Foundation, its role is described as mainly fundraising and long-term strategy, with limited involvement in the daily life of the community. It is also emphasized that though members of the Foundation may theoretically pull the switch if they completely disagree with the way the community is evolving, in practice dialogue is the way of solving various problems within the community. "I mean they could pay people to write stuff and so but it would not work, so I think the only way there is, besides just shutting the whole thing down, is to communicate and this is what is happening" (German Wikipedian \#6).

At a national level there is Wikimedia Germany, which is described as mostly administrative and deals with issues of fundraising, finances, contracts etc.

Practically all those interviewed emphasized that everything begins and ends with the community, thus this is the legitimate basis for defining and applying the rules guiding the daily practices within Wikipedia. It is thus the community that implements norms and rules each day as they edit and debate articles.

Policies are not developed in a (national) chapter, the policies are developed in communities [...]. The German chapter can take money and pay somebody and there the chapter comes into play and say okay the community wants this and that we will try to make it happen, but it is not a chapter that is pushing this because it does not work that way, it works the other way around (German Wikipedian \#6). 
Similarly, when asked about the development of new rules, it is stressed that most rules develop out of discussions within the community, rather than by some central mechanism. "It is my understanding there are some attempts to centralise, but usually these attempts do not lead to one centralised top for decision making, but to different roles that are at some point connected" (German Wikipedian \#5).

The German community is described as having comparably many rules, however as the rules may be ignored, they are not seen as indicative of a bureaucracy. The point that rules may be ignored refers to a specific norm within Wikipedia, the "ignore rules" norm, which imply that rules should not interfere with work on Wikipedia if they seem senseless. It is thus an encouragement to critical assessment if a rule seems to contradict a reasonable way of solving a problem.

If there is a rule that does not make any sense to you, please don't let this rule get in the way of your work! If you have the choice between not doing and sticking to the rule, and doing something and ignoring the rule, just do it anyway and deal with the consequences and if the rule is indeed stupid then well then you shouldn't have anything to worry about. $[\ldots]$ this corrective is highly efficient to prove structures to be ineffective because it helps people to get along with structures that no longer have any value. It is official, you can simply ignore any regulation if you feel it is stupid (German Wikipedian \#5).

The community rules are often bundled in guidelines and policies. With regard to content policies, the principles of "Neutral Point Of View", "Verifiability", and "No Original Research" are the main standards. As previously mentioned, the NPOV implies that all Wikipedia content must represent significant views fairly, proportionately, and without bias.. The Verifiability policy states that "material challenged or likely to be challenged, and all quotations, must be attributed to a reliable, published source". 9 The policy of No Original Research stresses that Wikipedia does not publish original thought, thus all material must be attributable to a reliable, published source. As previously discussed, the process of quality control is given much weight within the German community. However, the community also exhibits examples of less restrictive content regulation, compared to the English Wikipedia. For instance, uploading local images without any pointer to Wikimedia Commons is allowed.

With regard to hierarchy, the community distinguishes between "authors new to Wikipedia" and "editors in good standing". Only those who make enough edits to be considered active Wikipedians may participate whenever there is a vote within the community. As stressed by one of the interviewees, some make many edits to obtain this status (U.S. member of Wikipedia Advisory Board \#7) ${ }^{10}$. Further, the community has defined three levels of official positions; administrator, bureaucrat, and steward, which editors in good standing may apply for.

Administrators have the technical ability to delete and undelete pages, block and unblock users and IP addresses, protect and unprotect pages, and edit the interface, yet only on the local wiki where they are appointed. In the early years, being appointed admin- 
istrator was "no big deal" (James quoted in Lih, 2009, p. 95); however as the community grew, the processes around adminship formalized into a voting system with procedures for adminship nominations and approval. One of the interviewees stressed that the German procedure around adminship is increasingly complex with many votes and many internal conflicts (German Wikipedian \#1).

Bureaucrats, the second group of Wikipedia officials, are users with the technical ability to promote other users to administrator or bureaucrat, to grant and revoke a user's bot flag, and to rename a user.

\begin{abstract}
There are the bureaucrats [...], if you draw an organizational chart they are on top of it, but the truth is that they only got so much control because they don't really use it. They just use it in a way that some just, logical thinking person would use it, and if they would misuse it suddenly their power would be gone. So it is. Of course you can say they are on top of the hierarchy but the truth is that they are only there as long as they are doing what the collected will of the community wants, so I would not really call this a hierarchy. I think the hierarchy that is there is much more informal, is much more like okay this and this person have been active since 2003 and have written several dozen excellent articles and have been active in certain discussions and people agree that the things that this person says are very thoughtful and so on and this is what makes an important person in the community [...] (German Wikipedian \#6).
\end{abstract}

As articulated by the quote, the position of bureaucrat is not seen to imply power per se, but is indicative of community trust and respect for the work conducted by that particular person.

The top level of Wikipedia's official positions is the steward. Stewards are users with complete access to the wiki interface on all Wikimedia wikis, including the ability to change any and all user rights and groups, and to deal with emergencies and intervene against cross-wiki vandalism. They are elected by the global Wikimedia community, and appointed from the elected candidates by the Board of Trustees. Steward candidates need a support/ oppose ratio of at least $80 \%$ with at least 30 supporting users. ${ }^{11}$

When discussing with the interviewees the hierarchical structure outlined above, they all stress that the real power comes from the trust the community has toward a certain person, rather then the formal appointments. However, it is also argued that it is part of the culture to state that being an Admin, a Bureaucrat or a Steward is not a position of superiority. There is thus a sense of downplaying the role of these positions, when in reality most Wikipedians are proud of being appointed to, for example, an admin position, just as there is community status associated with these appointments.

Every administrator has the fact that they are administrator on their user page. I mean I have never met an admin who didn't.[...]. If you are getting into an argument with someone, it doesn't mean that you are going to win, but at least it's a proxy for the fact that a 
group of people have considered your contributions and validated them (U.S. member of Wikipedia Advisory Board \#7).

I mean to be an administrator maybe it is seen as a small sign which is recognisable from the outside. But it is much more what the community members think about the certain person which makes this person kind of a community leader (German Wikipedian \#6).

The emphasis on the community as "king" of Wikipedia is stressed time and again, and it seems fair to conclude that, according to the interviewees, the various positions are only worth something if backed by community trust and respect towards the individual. As such, the positions are not necessarily indicative of the most powerful people within Wikipedia, as power first and foremost derives from community recognition and respect, which is earned via the edits and arguments presented in the day-to-day work in the community. Other community tools related to problem notification and problem solving include the Administrator notice board and the Wikipedia counter-vandalism unit.

As outlined above, Wikipedia relies on its own community to identify and correct various problems that arise in relation to specific articles or users. While the open Wikipedia design provides an easy access to vandalise pages, it also provides a public track of the acts of vandalism, and easy access to rectify the articles in question. ${ }^{12}$ As a final resort for disputes, the community has installed an Arbitration Committee, which is a panel of editors responsible for conducting arbitration, with the authority to impose binding solutions to disputes between editors. Rulings enforced through the Arbitration Committee may result in a lifetime exclusion from Wikipedia and blocking by IP address or user name. ${ }^{13}$ The German community established an Arbitration Committee in 2007, and one of the interviewees stressed that this was to ensure a more legal process to handle conflicts. "We don't want decisions by mob rule, but more focus on judicial evidence" (German Wikipedian \#1). The arbitration mechanism is stressed as the main example of bureaucracy within the German community, however one that is legitimate (German Wikipedian \#4).

In summary, the community is self-organizing and with self-appointed public positions. Despite a relatively high number of rules and policies, the practical barriers to enter the community and to start contributing are low. Furthermore, everyone in principle has access to participate in governing Wikipedia after having earned some credits within the community. As such, access to power is generally available, however premised on being online and having achieved a certain level of recognition within the community. The German community is described as containing many rules and procedures, however it is not perceived as a bureaucracy, but rather a community guided by many rules, which one is somewhat free to ignore. The means of conflict resolution are largely based on dialogue, however votes are also often held, e.g., in relation to official positions. The community is stressed as being the "king" of Wikipedia, hence official positions first and foremost indicate that the individual has earned respect within the community. 


\section{Conclusion}

The aim of this study was to examine how a particular Wikipedia community reflects the values often associated with free cultures, such as openness and sharing.

The study largely confirmed the significance of openness and sharing amongst those interviewed, but also pointed to areas where the German community had deployed limits to openness. The study highlighted the strong focus on quality, implying that no article was better than a bad article. Moreover, the norm of dialogue is manifested in the numerous and lengthy debates amongst German Wikipedians in virtual as well as physical spaces. The frequent physical meetings are emphasized as an important feature of the community, not least as they may counter conflicts that would otherwise escalate online.

The norms of openness and sharing were visible in relation to the technical platform (open for all, no registration required), in relation to the legal license (sharing and reuse allowed), and in the cooperative practices. Compared to other voluntary associations, the Wikipedia norms of openness and sharing may not differ essentially, however the technical platform provides different means for realizing these norms. Likewise, the wiki platform facilitates a collective and creative space that is essentially different from non-mediated cooperation.

The study stressed that reactions from, and interactions with, fellow Wikipedians constituted one of the strongest motivational drivers amongst the interviewed. The interviewees are well aware of the normative goal of Wikipedia, however their daily practices seem less occupied with the general public or public life as such, and more oriented towards their own areas of interests, on-going debates within the community, and fellow Wikipedians. The study thus points to a relatively high degree of internal focus compared to an external focus, e.g., on the value of Wikipedia for its users, or for countries or regions with a less developed domain of public information.

As the study is based on interviews amongst a limited group of people, the findings should not be seen as representative of the 1.3 million German Wikipedia users, but rather as a qualitative testimony of the norms, values, and practices of the German Wikipedia community as it is perceived by some of the insiders from the Berlin Wikipedia community. As illustrated above, there are many dimensions of Wikipedia practices, and several aspects where these practices differ from what is often presumed about open cultures such as Wikipedia: for instance, the strong focus on quality, the high level of self-regulation, and the many arguments and conflicts. As such, the study does not point to homogenous or harmonious groups of people, but rather stresses the various means by which the community operates despite disagreements and harsh debates. Moreover, it stresses the complexity of reasons for taking part in Wikipedia. With the increasing focus on open cultures, it becomes important to understand not only the norms and practices of these communities, but also why people choose to take part in some, and not in others. In this particular study, the main motivation for participation was the sense of excitement and reward, which the interviewees experience when other people engage with their writing. Furthermore, the 
means of gaining visibility in front of a larger crowd, while remaining anonymous, was seen as fascinating.

\section{Notes}

1. The numbers refer to the list of interviewees in Jørgensen (2012, Appendix B).

2. Various statistics related to the German Wikipedia are available at: http://stats.wikimedia.org/EN/ ChartsWikipediaDE.htm and http://stats.wikimedia.org/EN/TablesWikipediaDE.htm, retrieved November 1, 2011.

3. See http://wikimania2005.wikimedia.org/, retrieved November 1, 2011.

4. See http://www.comscore.com/press/release.asp?press=2160, retrieved November 1, 2011.

5. See http://en.wikipedia.org/wiki/Wikipedia:Five_pillars, retrieved November 1, 2011.

6. See http://en.wikipedia.org/wiki/Wikipedia:Wikipedia_is_a_community, November 1, 2011.

7. See Lih (2009, pp. 122-132) for a detailed account of one of the edit wars regarding the article on the Polish city Gdansk, also known as Danzig.

8. Quote from http://meta.wikimedia.org/wiki/Wikipedia_power_structure, retrieved November 1, 2011.

9. Quote from http://en.wikipedia.org/wiki/Wikipedia:Verifiability, retrieved November 1, 2011.

10. A study on the criteria for promotion within Wikipedia (to become administrator, for example) suggests that there is a $10 \%$ increase in likelihood of Admin approval for every 3800 edits the individual has conducted (Burke \& Kraut, 2008).

11. See http://meta.wikimedia.org/wiki/Stewards, retrieved November 1, 2011.

12. Vandalism is defined as "any addition, removal, or change of content made in a deliberate attempt to compromise the integrity of Wikipedia". Common forms of vandalism include the addition of obscenities, page blanking, and the insertion of nonsense into articles. See http://en.wikipedia.org/wiki/ Wikipedia:Vandalism, retrieved November 1, 2011.

13. Research on the Arbitration Committee in the English edition finds that strict remedies are rarely used and that the Committee tries to encourage productive Wikipedians back into participating (Hoffman \& Mehra, 2009).

\section{References}

Benkler, Y. (2006). The Wealth of Networks - How Social Production Transform Markets and Freedom. New Haven, CT: Yale University Press.

Bruns, A. (2009). Blogs, Wikipedia, Second Life, and Beyond. From Production to Produsage. New York: Peter Lang Publishing.

Burke, M. and R. Kraut (2008). Mopping up: modelling wikipedia promotion decisions. Conference on Computer Supported Cooperative Work, San Diego: CA.

Hoffman, D.A. and S. Mehra (2009). Wikitruth Through Wikiorder. Emory Law Journal, Vol. 59, 2010.

Jørgensen, R.F. (2012). Framing the Net - How Discourse Shapes Law and Culture. Department of Communication, Business and Information Technologies. Copenhagen, Roskilde University. PhD.

Lessig, L. (2004). Free culture : how big media uses technology and the law to lock down culture and control creativity. New York: Penguin Press. 
Lih, A. (2009). The Wikipedia revolution: how a bunch of nobodies created the world's greatest encyclopedia. New York: Hyperion.

London, S. (1995). "Collaboration and Community." Retrieved July 12, 2011, from http://www.scottlondon. com/reports/ppcc.html.

McMillan, D.W. and D.M. Chavis (1986). Sense of community: A definition and theory. Journal of Community Psychology 14(1): 6-23.

On The Media (January 14, 2011). "10 Years of Wikipedia: Transcript." On the Media. Retrieved September 2, 2011, from http://www.onthemedia.org/2011/jan/14/10-years-of-wikipedia/transcript/.

Reagle, J.M. (2010). Good faith collaboration : the culture of Wikipedia. Cambridge, Mass.: MIT Press.

Schulz, W. and T. Held (2001). Regulated Self-Regulation as a form of modern Government. Study Commissioned by the German Federal Commissioner for Cultural and Media Affairs, Interim Report. Hamburg, Germany.

Stern.de (December 5, 2007). Wikipedia schlägt Brockhaus. Retrieved August 28, 2011, from http://www. stern.de/digital/online/stern-test-wikipedia-schlaegt-brockhaus-604423.html.

Sunstein, C.R. (2006). Infotopia : how many minds produce knowledge. Oxford: Oxford University Press.

Thagaard, T. (2004). Systematic approaches and Empathy. An introduction to qualitative methods. Copenhagen: Akademisk Forlag.

Zittrain, J. (2009). The future of the internet--and how to stop it. New Haven, Conn.: Yale University Press.

Rikke Frank Jørgensen

Researcher, Senior Adviser

Danish Institute for Human Rights, Denmark

rfj@humanrights.dk 